\title{
Technology and human purpose: the problem of solids transport on the Earth's surface
}

\author{
P. K. Haff \\ Nicholas School of the Environment, Division of Earth and Ocean Sciences, Duke University, Durham, North Carolina, \\ 27516, USA
}

Correspondence to: P. K. Haff (haff@ duke.edu)

Received: 1 May 2012 - Published in Earth Syst. Dynam. Discuss.: 24 May 2012

Revised: 11 September 2012 - Accepted: 19 October 2012 - Published: 14 November 2012

\begin{abstract}
Displacement of mass of limited deformability ("solids") on the Earth's surface is opposed by friction and (the analog of) form resistance - impediments relaxed by rotational motion, self-powering of mass units, and transport infrastructure. These features of solids transport first evolved in the biosphere prior to the emergence of technology, allowing slope-independent, diffusion-like motion of discrete objects as massive as several tons, as illustrated by animal foraging and movement along game trails. However, highenergy-consumption technology powered by fossil fuels required a mechanism that could support fast advective transport of solids, i.e., long-distance, high-volume, high-speed, unidirectional, slope-independent transport across the land surface of materials like coal, containerized fluids, minerals, and economic goods. Pre-technology nature was able to sustain regional- and global-scale advection only in the limited form of piggybacking on geophysical flows of water (river sediment) and air (dust). The appearance of a mechanism for sustained advection of solids independent of fluid flows and gravity appeared only upon the emergence of human purpose. Purpose enables solids advection by, in effect, simulating a continuous potential gradient, otherwise lacking, between discrete and widely separated fossil-fuel energy sources and sinks. Invoking purpose as a mechanism in solids advection is an example of the need to import anthropic principles and concepts into the language and methodology of modern Earth system dynamics. As part of the emergence of a generalized solids advection mechanism, several additional transport requirements necessary to the function of modern large-scale technological systems were also satisfied. These include spatially accurate delivery of advected payload, targetability to essentially arbitrarily located destinations (such
\end{abstract}

as cities), and independence of structure of advected payload from transport mechanism. The latter property enables the transport of an onboard power supply and delivery of persistent-memory, high-information-content payload, such as technological artifacts ("parts").

\section{Introduction}

Transport of mass and energy are among the most important processes displayed by dynamic systems. The nature and origin of large-scale transport processes for global Earth systems such as the hydrosphere, lithosphere, and atmosphere are basic ingredients for understanding how the Earth has evolved and how it functions. We understand the gross motions in these systems as responses to the availability of large quantities of high-quality energy (exergy) in the form of sunlight or the high-temperature internal heat source of the planet. (Here and below, unless otherwise indicated, "largescale", or "long" in reference to distance refers to regional, continental, or global scales - hundreds to thousands of kilometers - and "large", "large-scale" or "high" in reference to energy consumption derives from the terawatt scale of modern technological metabolism). In each case we can trace through the physical chain of events by which available energy generates forces that govern large-scale flows within these geologic paradigms. In recent Earth history a new Earth paradigm has emerged - the collection of interlinked systems that comprise regional- to global-scale technology. Largescale technology (or, for short, the "technosphere") is the term used here collectively for power, communication, industrial, governmental, military, and other widely distributed 
and interconnected technological systems on whose function modern civilization and society is based. Technology, including its human components, is recognized as an Earthchanging phenomenon whose transformation of air, water, and soil has been captured under the rubric of a new geologic epoch, the Anthropocene (Crutzen and Stoermer, 2000). Large-scale technology is largely powered by fossil energy sources, which in 2009 supplied more than $80 \%$ or $411 \mathrm{EJ}$ (International Energy Agency, 2011) of world total primary energy supply at a power level in excess of $10 \mathrm{TW}$. Unlike for the earlier Earth paradigms, in the case of the technosphere the mechanisms underlying energy flow from concentrations of free energy to waste heat cannot be analyzed by appeal solely to concepts of physics; human agency and purpose play a central role in many technological transport processes.

The production, movement, and consumption by the technosphere of energy, materials, and goods is accordingly often treated as an economic problem. Economics incorporates concepts and relationships such as supply and demand that directly relate human behavior to the flow of energy and mass through the technosphere. Among the goals of Earth science, on the other hand, is to understand the evolution of the planet as an organic system, including the rise of humans and technology as the most recent paradigm of Earth function, and to adopt into the science of Earth evolution an appropriate analytical framework based on these new emergent phenomena. Economics examines one side of this equation, emphasizing the role of humans. There are fewer investigations in which humans are explicitly treated as parts of the Earth system. Examples of work with the latter perspective are those of Werner and McNamara (2007) and Lazarus et al. (2011), in which the effects of geologic and social forces are tightly integrated, with landscape responding to human actions and humans in turn adjusting to landscape response. The present paper focuses on dynamics of mass and energy flows in the technosphere from a geological perspective, but adds one essential factor missing from classical geology purpose - and attempts to clarify the role that it plays in technospheric transport. Ultimately, purpose becomes necessary because of a singular physical fact - that the technological mass that undergoes transport (Haff, 2010) by the technosphere is mostly comprised of solid-like materials. By virtue of their intrinsic ability to store information without a power source, solids also form the essential substrate of technology. However, the same non-deformability that preserves information makes solids more difficult to transport for a given driving force than fluids, for which pre-technology Nature had evolved effective global transport mechanisms as evident in the hydrosphere and atmosphere. Prior to the emergence of human purpose Nature was unable to effect sustained largescale transport of solids, except those like river sediment that piggybacked on fluid flows.

In the following sections we discuss large-scale, e.g., regional to global, unidirectional displacement of solids (advection) as a fundamental transport mode of the technosphere, and compare with the localized (diffusion) modes, such as those operating at village scale, to which technological transport was limited prior to emergence of the technosphere. Basic scaling arguments, like those used in physics to distinguish between advective and diffusive phenomena in fluid transport (i.e., like the Reynolds number), are employed to compare different dynamical regimes of technological mass transport. Future investigations might consider hybrid, i.e., mixed-regime transport, such as that exemplified by Levy flights (Reynolds et al., 2007). Methods such as those that use cellular automata to mimic combined diffusive and advective motions of transported mass (Haff, 2001) also offer natural ways to extend the present scaling arguments. The relative effectiveness of advective and diffusive transport of mass can be compared through the metric of mass action (Haff, 2010) which weights the quantity of mass moved by a typical displacement distance and velocity.

Onboard power supply, rotary motion, and infrastructure are discussed as mechanisms that act to overcome retarding effects of frictional and form-resistance in solids transport. The onboard power supply is noted as a special case of independence of payload from transport mechanism, a property that enables transport and assembly of mass (i.e., solids) with arbitrary and enduring information content - a basic requirement of technological evolution. Purpose is then interpreted as the enabling mechanism by which information-rich mass is transported with high accuracy between two widely separated points despite the absence of an intervening potential gradient (whose presence would be the usual physical requirement for maintenance of energy and mass flows). Finally we note the challenge to Earth science of integrating into its narrative the role of teleological forces. It should be emphasized for clarity that, as used here, human purpose and teleology do not refer to any kind of force or process that lies outside physical law, but to emergent properties that are useful on their own terms for analyzing system behavior.

\subsection{Advective transport of solids}

Global technological function depends on rapid transport of solid-form energy resources like coal or containerized hydrocarbons and of solid-like materials, or "solids", of limited deformability, such as ores, sand and gravel, food, freight, people, as well as assembled or partially assembled subsystems or parts destined for integration into larger technological systems. Fossil fuel deposits and other mineral deposits tend, for geological reasons, to be distributed in zones of concentration that are widely dispersed across the globe. Sources of parts (factories) are also scattered geographically. Thus maintaining the metabolism of large-scale technology, i.e., the energy consumption of all technological processes, demands that the requisite solids be transported over long distances, up to thousands of kilometers, between discrete locations with high accuracy. 
The fast metabolism of modern technology $(\sim 13 \mathrm{TW})$ also requires high-speed (meters or tens of meters per second), high-volume (e.g., $\sim 5 \mathrm{GT}$ or $\sim 5 \mathrm{~km}^{3}$ of coal was consumed worldwide in 2008; World Energy Council, 2010) transport. In other words, an advective mechanism is needed to support the bulk transport of technological mass. This may be compared to a diffusion-like (referred to below for simplicity as "diffusive") transport mechanism in which small parcels of mass are moved about with frequent changes in direction of motion. In the lexicon of this paper diffusive movement of "small" parcels (e.g., a bottle of milk) could refer to dispersal by car, delivery trucks, and similar conveyances through city streets or to the movement of biomass by animals foraging for food, whereas transport over major long-distance highways or rail lines of bulk food represents an advective process. As an example, we consider a unit of mass transported over a straight-line distance $L$ at a given speed $v$ (advection), and compare with the same unit of mass transported over the same net distance at the same (instantaneous) speed $v$ but by means of a large number of small displacements $l \ll L$ that are quasi-randomly oriented with respect to one another (diffusion). If on dimensional grounds we define a mass diffusivity as the product $v l$ such that the diffusive timescale is $L^{2} / v l$, then the ratio of diffusive to advective time scales for transporting the mass the distance $L$ is $L / l$. If a fleet of trucks traveling at fixed speed on a highway that connects two cities that are $100 \mathrm{~km}$ apart requires $1 \mathrm{~h}$ to make the trip, the same trucks moving at the same speed on a random network of roads segments each with length $0.1 \mathrm{~km}$ (the length of a typical city block) would require on the order of $(100 / 0.1) \times 1 \mathrm{~h}$, i.e., $1000 \mathrm{~h}$, or almost six weeks, before a significant fraction of the trucks had moved $100 \mathrm{~km}$ from their starting point.

A metric that captures (i) the amount of mass moved, (ii) the distance that the mass moves between significant changes in direction (such as the highway or city-block distance measures of the preceding paragraph), and (iii) the speed of displacement, is mass action (Haff, 2010), $A=m l v$, where $m$ is the quantity of mass, $l$ is the displacement distance or mean free path, and $v$ is the average displacement velocity along $l$. When summed over the different masses moving in a given transport process, such as process A, highway traffic, or process B, city traffic, then the ratio of the summed mass actions for the two processes is the relative mass action number $\mathrm{Ma}_{\mathrm{A}, \mathrm{B}}=\sum_{\mathrm{A}} m l v / \sum_{\mathrm{B}} m l v$. (To make contact with a more familiar problem, if processes $\mathrm{A}$ and $\mathrm{B}$ refer to transport of molecules in a fluid by (A) eddies, and of the same molecules by (B) their thermal motion, then $\mathrm{Ma}_{\mathrm{A}, \mathrm{B}}$ reduces to the Reynolds number of the fluid, $\mathrm{Ma}_{\mathrm{A}, \mathrm{B}} \rightarrow R e$.) In the example of the previous paragraph, the mass action number for highway versus city street transport of the same mass at the same instantaneous speed would be in order of magnitude Mahighway,city $\sim L / l=1000$. The total mass action of technological land transport processes is of the same order of magnitude as the mass action associated with sediment transport by all the world's rivers (Haff, 2010).

Advection of solids over large distances occurs in nature where solids piggyback on fluid motion, as in the transport of river sediment. However, to support the high metabolic rate of technology that uses resources fluxed from random geographic locations, a mechanism is required that can transport solids across the Earth's surface in directions and at rates that do not necessarily have any connection to natural fluid flows. Sustained movement of large quantities of solids across the Earth's surface is difficult for several reasons. The first is that the friction coefficient between the land surface and most solid bodies is large. An irregularly shaped block of solid material will typically stay in place when positioned on a slope less than the angle of repose, whose value is usually on the order of 30 degrees (Anderson and Anderson 2010), implying a coefficient of friction $\mu \geq 0.6$. A solids transport mechanism in which the block was simply pushed across a horizontal surface would require a force $F_{\mathrm{p}}=\mu m g$, where $m$ is the mass of the block and $g$ is the acceleration of gravity. If for illustration we take $\mu=1$, then a $1 \mathrm{~kg}$ mass would require a minimum force of at least 10 newtons in order to keep it moving on level terrain. A block of coal with nominal mass density $\rho_{\text {coal }} \sim 1000 \mathrm{~kg} \mathrm{~m}^{-3}$ and linear dimension $D=0.1 \mathrm{~m}$ has a mass of $1 \mathrm{~kg}$ and a crosssectional area $A=0.01 \mathrm{~m}^{2}$. For a strong near-surface wind with speed on the order of $V_{\text {wind }}=10 \mathrm{~m} \mathrm{~s}^{-1}$, with air density $\rho_{\text {air }}=1 \mathrm{~kg} \mathrm{~m}^{-3}$ and aerodynamic drag coefficient $c \sim 1$, the force exerted on the block by the wind would be in order of magnitude $F_{\mathrm{w}} \sim A c \rho_{\text {air }} V_{\text {wind }}^{2}=1 N$. Thus the ambient power $F_{\mathrm{w}} V_{\text {wind }} \sim 10 \mathrm{~W}$ supplied by even a strong wind at the surface is too small to displace even a relatively low-density solid object of modest size. Small particles, for which the ratio of surface to body force is large, can be moved long distances by fluid forces, as happens in dust storms, or when sand and gravel are transported in a river, but until geologically recent times there existed no mechanism by which large amounts of solid mass could be moved unidirectionally across the land surface for a sustained period of time.

\section{Elements of solids transport}

\subsection{Onboard power source}

Aside from displacement by occasional events like landslides, transport across the land surface of solid or limiteddeformability objects as large as several tons became possible in Earth history (and common) only with the evolution of land animals. The appearance of tetrapods in the Middle to Late Devonian (Niedzwiedzki et al., 2010) (and earlier for arthropods) overcame the low ambient power density, highfriction limitations to solids transport on land by the introduction of two innovations, both of which later also played a critical role in enabling technological transport. The first 
innovation was inclusion of the motive power source as part of the transported mass. A self-powered human, weighing $50 \mathrm{~kg}$, walking on a level, firm surface at moderate speed $\left(1.3 \mathrm{~m} \mathrm{~s}^{-1}\right)$ requires an energy expenditure rate of about $60 \mathrm{~W}$ above base metabolic rate (Ainsworth et al., 1993), a level easily maintained for extended periods of time (hours). Selftransport by legged animals occurs mostly over relatively small distances with frequent changes in direction, i.e., it is diffusive. Nonetheless, the biological evolution of onboard power systems was an essential step in enabling land transport over a high-friction surface where reliable sources of ambient mechanical power were lacking. Technological transport on land faced the same power problem as biological transport, and adopted the same solution - onboard motors, such as those that propel cars, trucks, and trains.

\subsection{Rotary motion}

An onboard power source alone, however, is not sufficient to overcome frictional resistance to sustained motion either for biological or for technological systems. Using humans again as an example, the $60 \mathrm{~W}$ drawn from an internal energy source would not be very effective by itself in powering translation across the land surface if the transport mode were sliding. For a $50 \mathrm{~kg}$ person to drag herself (with $\mu=1$ ) across the ground at a typical walking speed would require the application of $\sim 500 N$ of force and an energy expenditure rate greater than $\sim 600 \mathrm{~W}$. A second transport innovation was required to support large-animal locomotion on land. This was rotary motion, which appeared in the form of pendulum-like oscillation of limbs in animals. Rotary motion significantly reduced the retarding effect of friction on dry land - in fact with rotation high friction becomes a virtue by reducing slippage - and enabled slope-independent diffusion of animals with mass measured in tons (e.g., mammals and dinosaurs). For the same reason rotary motion also emerged in technology, in the form of the wheel, enabling transport of multi-ton objects across high-friction terrain. Transport over rough terrain (as opposed to simply high-friction terrain) presented a different problem, discussed in the next section.

\subsection{Form resistance and infrastructure}

Even with an onboard power supply and a rotational mechanism that avoids the braking effect of friction, there remains a mechanical impediment to advective transport - what in fluid mechanics would be called form resistance. The flow of water in a river is driven by gravity and is resisted by two kinds of forces. The first is (skin) friction between the moving water and the bed. This frictional force is mediated by small-scale roughness of the bed and is analogous to the frictional resistance that is felt by a solid body dragged across a surface. Form resistance, or form drag (Furbish, 1997), on the other hand, arises from larger-scale surface roughness where the size of roughness elements may approach an appreciable fraction of the depth of flow. An analog to fluid form resistance is the impediment to motion offered a solid object on land by an obstacle with vertical dimension comparable to that of the object, or to parts of the object that are critical to the transport process, as a rock or shrub might offer resistance to the passage of an automobile or, with the same result, to its wheel. Form resistance to water flow across the Earth's surface is reduced by the spontaneous formation of a river channel, an emergent structure whose effect is to remove larger obstacles that might impede flow, as when a river cuts through rugged terrain and clears debris along the immediate flow path, and to decrease the relative size of surface irregularities in contact with advective flow (that is, to reduce the "wetted perimeter"). The river channel, in its modification of the transport environment to reduce form resistance, represents a kind of transport infrastructure. Advective transport of solids on land requires transport infrastructure like highways and railways for the same reason - to smooth the natural landscape.

\subsection{Independence of payload from transport mechanism}

The appearance of rapid long-distance solids transport made possible long-distance conveyance of payloads with arbitrary physical structure and properties. That is, (part of) the transported mass could be decoupled from the transport process itself. This is not the case with the Earth's other mechanism for large-scale movement of mass - fluid flow. The difference in transport mode of fluids and solids reflects differences in constraints on internal degrees of freedom of the transported mass. The easy deformability of fluids results in the accommodation of their internal configuration to the demands of transport. For example part of the mass of a river flowing over a rough bed is configured in a way that effectively smooths out surface irregularities, thus reducing friction for the rest of the flow, while the bulk of the flow configures itself into eddies that operate to transport momentum internally toward the bed as part of the transport process. In solids transport, by contrast, most internal degrees of freedom are uninvolved in the transport process - they are frozen in place and come along for the ride. Unlike in fluid flow, in solids transport there is a clear separation of "payload" from transport mechanism. There is then, in general, no necessary restriction for solids on the constitution of the payload.

\subsection{Solids and memory}

A significant consequence of decoupling of payload from transport mechanism follows from the fact that solids have enduring memory. Solids maintain their information content, i.e., the relative positions of their atomic constituents, during the transport process, whereas in contrast a purely fluid payload is not decoupled from its transport mechanism and must restructure on the fly as it participates in its own transport 
dynamics. A knife retains its sharp blade and a computer retains its transistors, keyboard and other elements of its design, and both retain their functionality, however far they are moved. An eddy in a river on the other hand does not maintain its structure. Preservation of memory during transport is a key property that allows construction of large technological systems from a collection of smaller technological parts or artifacts that are assembled at dispersed geographical locations following transport from the point of manufacture. This is a physical reflection of the combinatorial process that underlies technological evolution (Arthur, 2009). In-place construction of parts and subsystems that together comprise a large technological system is generally not possible because the distribution of source materials and information (knowledge) to be encoded in structure and function are determined by exogenous factors (like the distribution of geologic resources or human expertise) that bear no necessary relation to the disposition of the technology into which they will be incorporated. A transformer in an electrical distribution system does not grow organically in place but usually must be imported in one piece from the location where it was assembled. The information built into the transformer in the factory, and not otherwise available to the grid, is preserved in transit to become part of the total information content of the grid system. In a similar way all large technological systems are comprised of many composite solid parts, each with pre-installed memory, the overwhelming majority of which were advectively transported over large distances prior to incorporation as system parts. The importance of solids to memory in dynamical systems was first clearly stated by Schrodinger (1944) in his analysis of the stability of genetic information. His conclusions apply to information content in technology as well as in biology.

\subsection{High accuracy transport to arbitrary destinations}

A portable power source together with transportation infrastructure provides the basis for another property necessary to the function of spatially extensive, high-metabolism technologies - namely, high spatial accuracy of delivered payload to essentially any geographic location. Large technological systems contain parts and require resources whose accurate delivery and precise placement relative to other parts is critical for system function. For example, coal from a mine must end up at the power plant that burns the coal even though the power plant has no necessary geographical relation to the mine and may lie at a great distance from it. The Scherer power plant in Atlanta (McPhee, 1997) is a tiny target, subtending an angle of only 0.04 degrees as seen from its coal source at the Black Thunder Mine in Wyoming, but the coal nonetheless arrives exactly at the location of the power plant. Likewise food stuffs transported from the farm or from food processing centers must be targetable from a distance to specific supermarkets, and similar requirements of spatial resolution are present in supplying raw materials and parts to any manufacturing process. The intrinsic shape-stability of solids (a brick placed on the ground retains its compact shape while a similar volume of water immediately spreads out in a thin sheet unless confined by solid boundaries, as in a puddle) means that a suitable transport path can be accommodated by a narrow infrastructure zone. For example, the aspect ratio of the travel surface of a major transcontinental highway is typically about $10^{5}$. Transport corridors are thin, presumably because infrastructure construction and maintenance costs energy. Given that targeted transport is a function of purpose (see below), building wider-than-necessary infrastructure elements would represent a purposeless use of energy, and lowaspect ratio transport corridors would be the exception rather than the rule.

\section{Absence of continuous potential gradient}

In order for transport to persist, a driving force or affinity must do work to maintain the flow of mass, and to form and maintain infrastructure. For natural Earth systems the origin of the driving force is a gradient in an environmental variable, such as elevation in the case of a river. For the river we understand the physical processes that act to overcome friction and generate the infrastructural channel. These processes are direct consequences of extraction of energy from the gravitational potential and the consequent application of a force that causes flow and generates infrastructure via erosion and sediment transport (e.g., Anderson and Anderson, 2010). Sustained advection of solids across the Earth's surface must likewise be driven by a gradient in a suitable environmental variable. The oil pools and coal beds sequestered below the Earth's surface define an average gradient in stored chemical energy relative to any point where such deposits are scarce. However, unlike the space-filling gradient of elevation that at most points on the land surface provides a force that can power river flow, the chemical energy in fossil energy deposits is confined to the geologic source area and exerts no direct intervening influence by which to power motion of mass to distant points. This is one reason that fossilfuel deposits have not already undergone wholesale depletion. Some depletion does occur naturally of course, as in coal-bed fires (Heffern and Coates, 2004) or biodegradation of crude oil (Jones et al., 2008), but this tends to occur in situ and to be slow compared to the rates of technological energy extraction.

The energy associated with a step-function gradient, defined by a localized spatial distribution of the energy source, can sometimes be accessed by rearrangement of system parts, as when gas molecules arrange themselves in such a way as to smoothly interpolate a local temperature difference between separated sources and sinks of thermal energy. Heat can then be conducted down a continuous temperature gradient that the molecules themselves act to define. In the next section we discuss a somewhat similar kind of interpolation 
process that occurs with respect to the step-function distribution of fossil-fuel sources.

\section{Purpose and transport}

To help focus on a key factor necessary to understand the origin of advective solids transport, we first consider the example of atmospheric convection. In the temperature inversion that occurs when solar radiation heats the ground and adjacent layers of the atmosphere, the temperature difference between lower and upper atmospheric layers generates density differences and hence a buoyancy force that propels parcels of hot air upward as convective plumes. At each point in the motion of a typical ascending parcel of air, local pressure differences overcome the viscous (frictional) retarding force and sustain the parcel motion. Transport of an element of mass across the Earth's surface similarly requires continuous application of a suitable force to overcome friction. In the case of rail transport of coal from its source at a mine to the point where it will be consumed, the physical force that drives mass transport and overcomes retarding effects such as those offered by air resistance and internal friction is the force exerted by the rails on the wheels of the locomotive. The force is applied at every point along the transport path, or else the train would stop. Identifying this force is as full an answer to the question of what causes advection of coal as the identification of the buoyancy force answers the question of what causes hot air to rise. A fuller explanation of each phenomenon requires answering a different question, namely, what is the origin of the applied force? Because we can easily imagine how thermal conduction heats a layer of near surface air and because we have a physical understanding of why heated air expands in volume, and because we understand the basic physics of buoyancy, the explanation of thermal convection of the atmosphere can be made entirely in the language of physics.

In the example of transport of coal, while we can explain the physics of the force that causes the coal to advect in terms of the action of a diesel engine and all the internal linkages that end up exerting a force on the rail and then a force back on the train, there is no explanation in the language of physics for the origin of the force. Rather, the origin is explained as a consequence of human purpose: people want the coal to appear in a place that is distant from its source location. The dynamical problem that "purpose" solves is this: it enables dynamics that are capable of inducing transport even in the absence of a continuous potential gradient between separated source and sink zones. Purpose provided a basis for emergence of a nonlocal Earth system dynamics, in which mass and energy could propagate between widely separated points in the absence of an intervening force field. Human purpose is an ultimate cause of transport and the diesel engine is a proximate cause. The distinction between proximate and ultimate cause is familiar in biology (Mayr, 1961) where for example the proximate cause of the bird taking flight is the approach of a predator and the ultimate cause is a genetically determined survival instinct. The concepts embodied in Darwinian evolution provide an overarching theory of biological systems and the origin of certain ultimate causes. Construction of a comparable metatheory of technology has begun (e.g., Arthur, 2009), but is not yet well developed. Our understanding is still largely stuck in the "organismal" phase of technology where the focus is on materialized artifacts and systems rather than on the collective dynamics of the technosphere. Understanding human purpose and its effects as a product of Earth system evolution rather than as an independent phenomenon somehow springing full-blown from the individual human will be a sign of maturity in the development of a self-consistent theory of technology. At present we lack this understanding, and are forced to treat human purpose as an ad hoc element of technological dynamics.

Invoking human purpose as the cause of a physical force may seem like an abandonment of the principles of science, which, since Darwin, has mostly abjured teleological explanations (e.g., Mayr, 1992). There are good reasons for this stance - experience shows that physics, chemistry, and most of biology are explicable in terms that have nothing to do with human intention. However, it is also true that explanation of the behavior of any sufficiently complex system requires appeal to emergent phenomena or properties suggested by observation of the system itself and not (only) by reference to the underlying physics (Werner, 2003). The behavior of an ant colony is explained (Holldobler and Wilson, 1990) in terms of the actions or behavior of the queen, of drones and workers, of chemical trails and external environmental factors, and so on, not in terms of the underlying molecular physics. Physics and chemistry can inform our understanding of specific mechanisms of complex system function, such as the role of pheromones in ant signaling, but fail to provide a complete explanatory basis for whole system behavior. New levels of explanation are required. Human purpose, a product of Earth evolution, is an emergent real-world phenomenon that has physical effects, one of which is its role in enabling solids transport on land.

\section{Summary and conclusion}

Certain fundamental physical requirements had to be met for large-scale systems of the kind that dominate technological energy consumption today to emerge. One of these, studied here, was the capability for rapid transport of large quantities of materials of limited deformability, such as coal, minerals, and other products, across the land surface over large distances, i.e., advective transport of solids. The antecedents and path of development of today's multifaceted technology is often considered to lie in the bailiwick of historians (Basalla, 1989) and social scientists (Heilbroner, 1967), but one might expect that basic underlying functionality such as 
mass transport and energy flows would fall squarely within the bounds of the physical sciences. However, the mechanism of large-scale extraction of work from concentrated deposits of fossil chemical energy - the energetic basis of modern technology - is not entirely a problem of mechanics or thermodynamics. Consumption of these energy sources had to await the emergence of human purpose, which (effectively) provided for an otherwise missing potential gradient between fossil energy sources and sinks. When Earth evolution produced a mechanism that could provide support for such a transport mode, several further physical processes became possible that were also essential for the emergence of large-scale development of technology, including transport of complex payloads with persistent memory, displacement of these payloads independent of geophysical fluid flows and topographic slope, and spatially accurate delivery to fixed but arbitrary destinations.

The example of solids transport illustrates one way in which the dynamics of the Earth system today depends not just on variables familiar from the physical sciences but also on new emergent phenomena - in this case human purpose. Disciplines outside the physical sciences have arisen to engage the implications of purpose. For example, economics offers a set of well-developed rules that addresses energy- and mass-flow phenomena using purpose-based concepts such as supply and demand. However, to follow this example, economics alone is not a sufficient basis on which to explain the dynamics of the technosphere because, like other social sciences, it is partly a product of what it would be trying to explain. The principles of economics do not effectively account for the fact that economics itself is not a free-floating invention of humans, but a product of Earth system dynamics. We have the example of the biosphere as a set of complex systems - organisms and ecosystems - whose origin and development can be understood in terms of a metatheory, Darwinian evolution, whose basic elements (replication, variation and selection), considered as processes, not as realizations, are independent of biology. In this way we understand biology not as an add-on but as an integral part of the Earth system. On the other hand technology is usually considered a human-generated add-on to nature, its large-scale operation explainable at least in principle by engineering, economics, and other social sciences. Although this is clearly an incomplete assessment of what large-scale technology really is, at present there is no overarching framework for understanding the technosphere as an expression of Earth dynamics. The Principle of Maximum Entropy Production (so far still a conjecture) has been discussed (e.g., Kleidon and Lorentz, 2005) as one approach for analyzing energy consumption in essentially arbitrary complex systems subject to strong (enough) forcing, but the application of PMEP to the technosphere remains unproven and untested (Haff, 2012). Another approach might extend the Darwinian triumvirate of replication, variation, and selection to the technological world, as Blackmore's (2010) notion of techno-memes, or "temes" does, according to which technology would evolve for the same non-biological reasons that the biosphere evolves. However, no accepted technospheric dynamics has yet been constructed based on these ideas. The present study does not break this impasse, but attempts to move the discussion forward by emphasizing one example of how purpose, technology, and the larger Earth system all come together to enable the apparently simple physical process of large-scale transport of solid materials.

Acknowledgements. I thank Evan Goldstein, Brad Murray, Dave Furbish and an anonymous referee for suggestions for improving this paper.

Edited by: A. Kleidon

\section{References}

Ainsworth, B. E., Haskell, W. L., Leon, A., Jacobs Jr., D. R., Montoye, H. J., Sallis, J. F., and Paffenbarger Jr., R. S.: Compendium of physical activities: classification of energy costs of human physical activities, Med. Sci. Sport Exer., 25, 71-80, 1993.

Anderson, R. S. and Anderson, S. P.: Geomorphology: The Mechanics and Chemistry of Landscapes, Cambridge University Press, Cambridge, UK, 2010.

Arthur, W. B.: The Nature of Technology: What It Is and How It Evolves, Free Press, NY, 2009.

Basalla, G.: The Evolution of Technology, Cambridge University Press, Cambridge, UK, 1989.

Blackmore, S.: Temes: An emerging third replicator, in On the Human (National Humanities Center), available at: http: //onthehuman.org/2010/08/temes-an-emerging-third-replicator/, last access: 9 September 2012.

Crutzen, P. J. and Stoermer, E.: The Anthropocene, Global Change Newsletter, 41, 17-18, 2000.

Furbish, D. J.: Fluid Physics in Geology, Oxford University Press, New York, 1997.

Haff, P. K.: Waterbots, In Landscape Erosion and Evolution Modeling, edited by: Harmon, R. S. and Doe III, W. W., 239-275, Kluwer Pub., New York, 2001.

Haff, P. K.: Hillslopes, rivers, plows, and trucks: mass transport on Earth's surface by natural and technological processes, Earth Surf. Proc. Land., 35, 1157-1166, doi:10.1002/esp.1902, 2010.

Haff, P. K.: Maximum entropy production by technology, in: Beyond the Second Law: Entropy Production and Non-Equilibrium Systems, edited by: Dewar, R. C., Lineweaver, C., Niven, R. K., and Regenauer-Lieb, K., Springer, in press, 2012.

Heffern, E. L. and Coates, D. A.: Geologic history of natural coalbed fires, Powder River basin, USA, Int. J. Coal Geo., 59, 25-47, 2004.

Heilbroner, R. L.: Do Machines Make History?, Technol. Cult., 8, 335-345, 1967.

Holldobler, B. and Wilson, E. O.: The Ants, Harvard University Press, Cambridge, 1990.

International Energy Agency: Key World Energy Statistics, http: //www.iea.org/textbase/nppdf/free/2011/key_world_energy_stats. pdf (last access: 30 April 2012), 2011. 
Jones, D. M, Head, I. M., Gray, N. D., Adams, J. J., Rowan, A. K., Aitken, C. M., Bennett, B., Huang, H., Brown, A., Bowler, B. F. J., Oldenburg, T., Erdmann, M., and Larter, S. R.: Crudeoil biodegradation via methanogenesis in subsurface petroleum reservoirs, Nature, 451, 176-180, doi:10.1038/nature06484, 2008.

Kleidon, A. and Lorenz, R. D. (Eds.): Non-Equilibrium Thermodynamics and the Production of Entropy: Life, Earth, and Beyond, Springer Verlag, Heidelberg, 2005.

Lazarus, E. D., McNamara, D. E., Smith, M. D., Gopalakrishnan, S., and Murray, A. B.: Emergent behavior in a coupled economic and coastline model for beach nourishment, Nonlinear Proc. Geoph., 18, 989-999, 2011.

Mayr, E.: Cause and effect in biology, Science, 134, 1501-1506, 1961.

Mayr, E.: The Idea of Teleology, J. Hist. Ideas, 53, 117-135, 1992.

McPhee, J.: Uncommon Carriers, Farrar, Straus and Giroux, New York, 1997.

Niedzwiedzki, G., Szrek, P., Narkiewicz, K., Narkiewicz, M., and Ahlberg, P. E.: Tetrapod trackways from the early Middle Devonian period of Poland, Nature, 463, 43-48, doi:10.1038/nature08623, 2010.
Reynolds, A. M., Smith, A. D., Reynolds, D. R., Carreck, N. L., and Osborne, J. L.: Honeybees perform optimal scale-free searching flights when attempting to locate a food source, J. Exp. Biol., 210, 3763-3770, doi:10.1242/jeb.009563, 2007.

Schrodinger, E.: What is Life, The Physical Aspect of the Living Cell, 1st Edn., Cambridge University Press, 1944.

Werner, B. T.: Modeling landforms as self-organized, hierarchical dynamical systems, in: Prediction in Geomorphology, edited by: Wilcock, P. R. and Iverson, R. M., Geophysical Monograph Series, American Geophysical Union, Washington, DC, 135, 133150, doi:10.1029/135GM10, 2003.

Werner, B. T. and McNamara, D. E.: Dynamics of coupled humanlandscape systems, Geomorphology, 91, 393-407, 2007.

World Energy Council: Survey of World Energy Sources, World Energy Council, London, available at: http://www.worldenergy.org/ documents/ser_2010_report_1.pdf (last access: 30 April 2012), 2010 . 